\title{
A multi-objective approach to select hydrological models and constrain structural uncertainties for climate impact assessments
}

\author{
Danny Saavedra ${ }^{1}$, Pablo Mendoza ${ }^{1}$, Nans Addor $^{2}$, Harold Llauca $^{3}$, and Ximena Vargas ${ }^{1}$ \\ ${ }^{1}$ Universidad de Chile \\ ${ }^{2}$ University of Exeter \\ ${ }^{3}$ Servicio Nacional de Meteorología de Hidrología del Perú
}

June 25, 2021

\begin{abstract}
The assessment of climate change impacts on water resources and flood risk is typically underpinned by hydrological models calibrated and selected based on observed streamflow records. Yet, changes in climate are rarely accounted for when selecting hydrological models, which compromises their ability to robustly represent future changes in catchment hydrology. In this paper, we test a simple framework for selecting an ensemble of calibrated hydrological model structures in catchments where changing climatic conditions have been observed. We start by considering 78 model structures produced using the FUSE modular modelling framework and rely on a Pareto scheme to select model structures maximizing model efficiency in both wet and dry periods. The application of this approach in three case study basins in Peru enables the identification of structures with good robustness, but also good performance according to hydrological signatures not used for model selection. We also highlight that some model structures that perform well according to traditional efficiency metrics have low performance in contrasting climates or suspicious internal states and fluxes. Importantly, the model selection approach followed here helps to reduce the spread in precipitation elasticities and temperature sensitivities, providing a clearer picture of future hydrological changes. Overall, this work demonstrates the potential of using contrasting climatic conditions in a multi-objective framework to produce robust and credible simulations, and to constrain structural uncertainties in hydrological projections.
\end{abstract}

\section{Hosted file}

Saavedraetal2021HP_subm0.docx available at https ://authorea.com/users/421831/articles/527693a-multi-objective-approach-to-select-hydrological-models-and-constrain-structuraluncertainties-for-climate-impact-assessments 

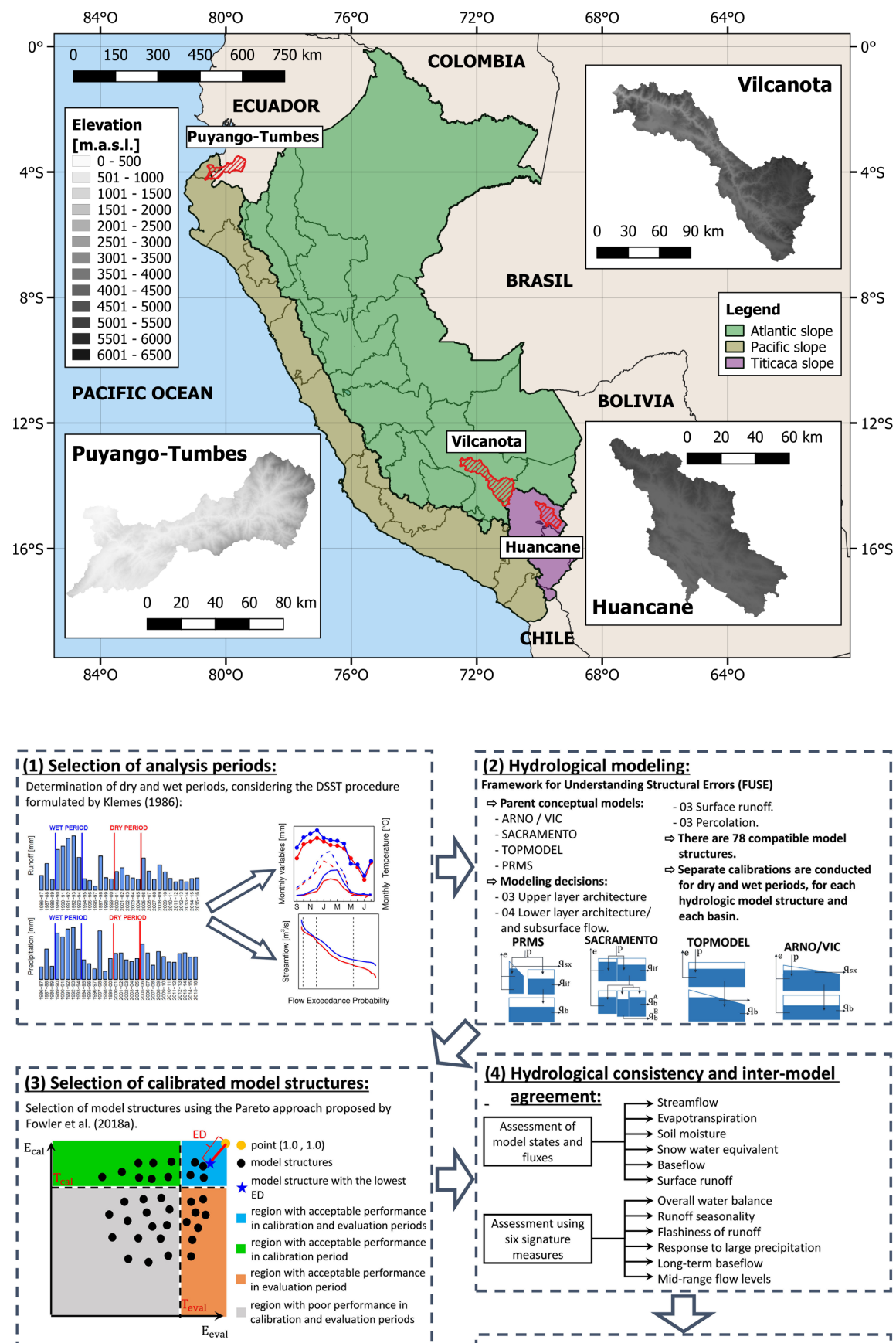

(3) Selection of calibrated model structures: I Selection of model structures using the Pareto approach proposed by
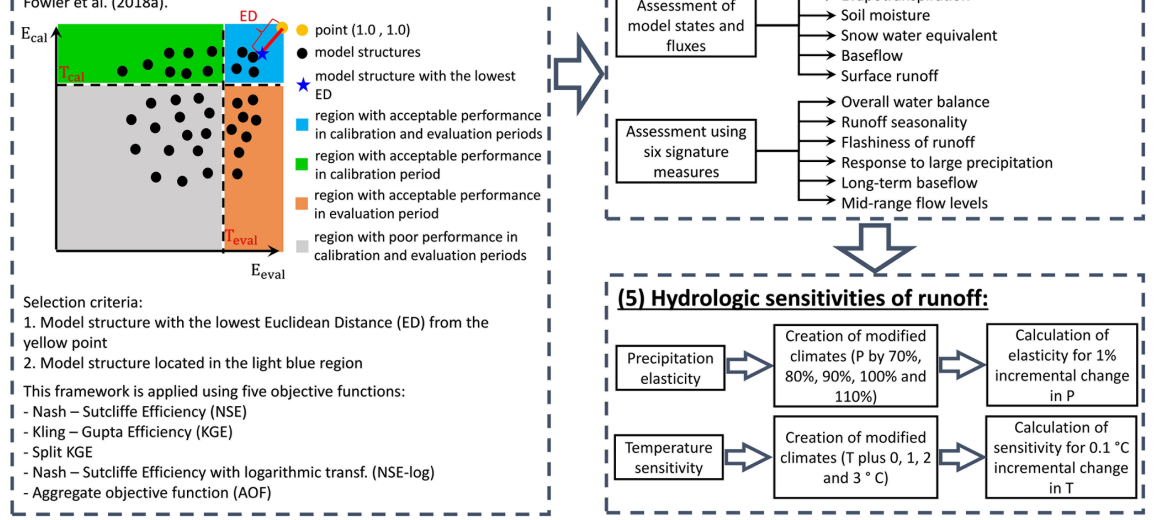
figures/Figure3/Figure3-eps-converted-to.pdf 
figures/Figure4/Figure4-eps-converted-to.pdf 
figures/Figure5/Figure5-eps-converted-to.pdf

\section{Hosted file}

Figure6.eps available at https://authorea.com/users/421831/articles/527693-a-multiobjective-approach-to-select-hydrological-models-and-constrain-structural-uncertaintiesfor-climate-impact-assessments 
figures/Figure7/Figure7-eps-converted-to.pdf 
figures/Figure8/Figure8-eps-converted-to.pdf 
figures/Figure9/Figure9-eps-converted-to.pdf 